\title{
In Memoriam: Hélène Brodeur
}

\author{
Beatriz MANGADA \\ Universidad Autónoma de Madrid \\ Departamento de Filologías y su didáctica \\ beatriz.mangada@uam.es
}

Recibido: 22 de octubre de 2010

Aceptado: 20 de diciembre de 2010

\section{RÉSUMÉ}

Hélène Brodeur (1923-2010), romancière emblématique de la littérature franco-ontarienne des années 80 , nous quittait en août 2010. Après une première approche contextuelle de la présence des grands espaces dans la littérature franco-canadienne en tant que lieu de rencontre d'identités spatiales en évolution, nous proposons une étude de la dérive énonciative autour de la dimension spatiale dans la trilogie d'Hélène Brodeur, Chroniques du Nouvel-Ontario. Nous voulons ainsi lui rendre hommage et contribuer à mieux comprendre son apport décisif à la consolidation de l'identité de la communauté franco-ontarienne, grâce à son témoignage narratif sur l'histoire de la colonisation du Grand Nord ontarien.

Mots clés: Hélène Brodeur, grands espaces canadiens, littérature franco-ontarienne.

\section{In Memoriam: Hélène Brodeur}

\section{RESUMEN}

Hélène Brodeur (1923-2010), escritora emblemática de la literatura franco-ontariense de los años 80, fallecía en agosto del 2010. Tras un primer acercamiento contextual a la presencia de los grandes espacios en la literatura franco-canadiense como lugar de encuentro de identidades espaciales en evolución, proponemos un estudio de la deriva enunciativa en torno a la dimensión espacial en la trilogía de Hélène Brodeur, Chroniques du Nouvel-Ontario. Queremos de este modo rendirle homenaje y contribuir a una mayor comprensión del decisivo aporte que supone su testimonio narrativo sobre la historia de la colonización del Gran Norte de Ontario en la consolidación de la identidad de la comunidad franco-ontariense.

Palabras clave: Hélène Brodeur, grandes espacios canadienses, literatura franco-ontariense.

\section{In Memoriam: Hélène Brodeur}

\begin{abstract}
Hélène Brodeur (1923-2010) was undoudtedly one of the most distinguished Franco-Ontarian writers. Our critical study of open spaces in her trilogy Chroniques du Nouvel-Ontario, published in the 1980s, will not only serve as a tribute to Brodeur, but will also elucidate her decisive contribution to the Franco-Ontarian emergent identity. Her narrative offers invaluable testimony of the colonization of Northern Ontario, and thereby promotes and enriches the debate about Canadian peripheral literatures, and their relation to vast geographical spaces.
\end{abstract}

Key words: Hélène Brodeur, Canadian Great Spaces, Franco-Ontarian Literature. 


\section{Les grands espaces dans la littérature franco-canadienne: lieu de rencontre d'identités spatiales en évolution.}

Lieu de rencontre de perceptions et de représentations spatiales en évolution, la description des grands espaces dans les différentes manifestations culturelles canadiennes, apparaît comme l'un des meilleurs moyens d'étudier l'évolution identitaire de cette communauté vis-à-vis de l'espace naturel. Dans un pays où environ $25 \%$ du sol est recouvert de vastes forêts et où les conditions climatiques et les nombreux et puissants cours d'eau réduisent les terres cultivées à $7 \%$, on peut facilement comprendre l'influence que ces immenses étendues de terres vierges ont eue sur ses premiers habitants et comment elles continuent à conditionner la vie de sa population actuelle. L'étude de l'expression du vécu spatial concerne non seulement géographes, mais aussi peintres et critiques littéraires. En effet, dans son article «La peinture québécoise d'Entre-deux-guerres» (Trepanier, 1996), l'auteure rappelait que chez les peintres canadiens des années 30 et 40 , aussi bien francophones qu'anglophones, s'était produite une transformation et remise en cause de la représentation visuelle de l'espace canadien. D'ailleurs, pour le géographe Gilles Sénécal, le rapport entre territoire, identité et imaginaire spatial passerait par une conception de territorialité qui n'est pas éternelle, et par conséquent, se suivent des phases de déterritorialité et de reterritorialité (Sénécal, 1992: 30). Il coïncidait également avec un autre géographe, A. Gilbert, pour qui l'analyse du discours permet de rendre compte des lieux en privilégiant les représentations, les sens et la manière de structurer l'espace (Gilbert, 1986).

Porté au domaine de la littérature canadienne, ce champ de travail a fait l'objet de nombreuses réflexions, recueillies entre autres dans Treasures of the place, three centuries of Nature writing (Grady, 1992) et $A$ few acres of snow, literary and artistic images of Canada (Simpson-Housley and Norcliffe, 1992), parmi les Anglophones et Espace et culture (Courville et Séguin, 1995) ou encore L'espace canadien et ses représentations (Guillaume, Lerat et Piccione, 1996), chez les Francophones. Sans oublier, bien entendu, la pensée du critique canadien, Ronald Bordessa, dont les lignes reproduites ci-dessous résument cette conception de l'espace comme attrait de nombreux artistes canadiens:

Canadian literature has greatly concerned itself with questions of identity. It is hardly surprising (if Frye has correctly specified the peculiarity Canadian riddle) that it has elaborated these questions in spatial terms. Identity is referential: in the European case, to time (genealogy, heritage, inheritance); in the Canadian case, to space (exploring, settling and transforming). The land and the landscape are metaphor for Canada and central preoccupations of its writer (Bordessa, 1992: 63).

Quelques pages plus loin nous lisons:

Landscape is not an external objective reality to be written, but a defining ingredient of the constitution of the Canadian experience. It is a condition of being, and post-modern literature consciously elaborating the experience of being can be expected therefore to be writing from landscape - rather than about landscape - because it is in our being (Bordessa, 1992: 67). 
Ce raisonnement nous rappelle de nombreux passages descriptifs de la littérature franco-canadienne qui illustrent un imaginaire américain au sens continental, à la recherche de soi à travers les grands espaces (Cubeddu, 2006).

Nous proposons par la suite un bref parcours à travers cette quête d'identité spatiale et sa projection littéraire. Notre étude démarre avec la période d'appréhension du nouveau continent de la part des premiers colons européens vers la deuxième moitié du XIXe siècle. Des écrivains tels que Patrice Lacombe avec Terre paternelle (1846), Pierre-Olivier Chaveau et Charles Guérin (1853) ou encore Antoine Gérin-Lajoie et son Jean-Rivard le défricheur (1862), nous parlent de l'immensité des espaces, de la rigueur extrême des hivers, toujours blancs et trop longs, et d'une nature qu'il faut combattre pour pouvoir y établir une société rurale stable et sédentaire; une société conçue de façon binaire, d'une part par des coureurs de bois et des trappeurs nomades, et d'autre part par des agriculteurs et des fermiers sédentaires. Or, l'expression de cette société naissante et son rapport à l'espace environnant trouve un plus grand développement dans les productions littéraires des années 1900 à 1930 . Selon le critique et grand connaisseur de la littérature québécoise Maurice Lemire, la défense de la terre commence au cours de cette période, bien que le terme «régionaliste» appliqué au roman canadien de cette époque acquiert un sens bien différent du roman régionaliste français (Lemire, 1980: xxiii-xxv). Tandis qu'en France ce genre braquait ses intérêts sur la description de la vie champêtre, au Canada, des écrivains comme Louis Hémon avec Maria Chapdelaine (1916), Léo-Paul Desrosiers avec Nord-Sud (1931) ou Félix-Antoine Savard dans son Menaud, maître-draveur (1937), tenteront d'exprimer à travers leurs perceptions spatiales le rapport toujours difficile entre le pionnier et le nouveau continent. Ainsi, ces écrivains «régionalistes» vont devenir les précurseurs d'une littérature canadienne différente qui cherchera l'expression de sa propre identité à travers un imaginaire qui lui soit également propre. Cette circonstance explique sans doute le recours à cet appel des grands espaces (Lemire, 1996) chez un grand nombre d'auteurs; or, Lemire ne cesse de rappeler que cette thématique caractéristique de l'imaginaire canadien n'a pas été malheureusement exploitée à temps, ce qui justifie que, si bien la figure de «voyageur» et l'appel des grands espaces atteignent leur pleine expression au XXe siècle, le décalage temporel résulte insurmontable entre le vécu qui l'inspire et le quotidien des lecteurs (Lemire, 1996: 72). Voici comment exprimait le narrateur de Maria Chapdelaine, cet abyme entre l'esprit sédentaire des fermiers et le voyageur toujours nomade, et qui nous rappelle sans doute une expérience dichotomique de l'espace:

C'était l'éternel malentendu des deux races : les pionniers et les sédentaires, les paysans venus de France qui avaient continué sur le sol nouveau leur idéal d'ordre et de paix immobile et ces autres paysans, en qui le vaste pays sauvage avait réveillé un atavisme lointain de vagabondage et d'aventure (Hémon, 1954: 47).

Ces «deux races» d'Hémon renvoient d'ailleurs à une conception binaire de l'espace proche du topos descriptif défendu par Philippe Hamon (Hamon, 1993: 205-239). En effet, aux pionniers nomades correspondrait une image de l'espace 
ouvert, immense, propre à l'esprit de conquête et de liberté qui, cependant, est mal vu aux yeux des sédentaires, car, pour ceux-ci, l'espace symbolise les fermes et les petits villages, synonymes de civilisation, de vie aisée propre aux référents européens. L'espace, une fois dompté, agira désormais en tant que garant du maintien des valeurs traditionnelles telles que la famille, la langue française et la vie religieuse.

$\mathrm{Au}$ cours des années 50, la nature cesse d'être cet élément hostile qu'il faut combattre pour acquérir, entre autres, l'apparence et la signification des plaines du Manitoba que Gabrielle Roy a su si bien «peindre» dans ses romans; finalement, un refuge qui permet de fuir les grandes villes émergentes et que nous retrouvons entre autres dans Bonheur d'occasion (1945) ou La route d'Altamont (1955). Le regard des personnages devient alors un médiateur capable de véhiculer les pensées et les états d'âmes évoqués par la contemplation des grandes étendues du Manitoba, de sorte que les personnages, transformés en voyeurs-médiateurs (Hamon, 1993: 212), vont déclencher la connotation de l'objet appréhendé dans leur perception. Dans son ouvrage Gabrielle Roy et Margaret Laurence: deux chemins, une recherche (Hughes, 1987), Terrance Hughes consacrait un chapitre à étudier la présence de l'espace chez ces deux écrivaines en mettant très spécialement l'accent sur la fascination qu'ont suscité les «immenses ciels du Manitoba» chez les personnages de Gabrielle Roy. Mais le Manitoba c'est surtout la plaine, les vastes prairies solitaires, comme celles dépeintes dans le fragment que nous reproduisons par la suite:

La plaine alors, me disait-elle, paraissait encore plus immense qu'aujourd'hui, et le ciel, lui aussi, plus immense; [...] j'étais attirée, avouait maman, penchant un peu la tête, comme s'il y eut un peu de mal à cela, tout au moins trop d'étrangeté. Attirée par l'espace, le grand ciel nu, le moindre petit arbre qui se voyait à des milles en cette solitude. J'étais très attirée (Roy, 1966: 163).

Hughes a recueilli également de nombreuses désignations contingentes $^{1}$ qui jalonnent l'œuvre romanesque de Gabrielle Roy et qui offrent une vision très particulière du Manitoba: de grands espaces, toujours immenses, recouverts de forêts et entourés de plaines et d'eau: Le Manitoba... paraissait alors s'ennuyer. Si grand, si peu couvert de noms, presque entièrement livré à ces larges étendues dépouillées qui figuraient les lacs et les espaces inhabités! (Roy, 1980: 152-153). Ou encore :

1 Rappelons, à cet égard, que le phénomène de l'anaphore a été largement abordé par la linguistique textuelle. Or, c'est surtout Francis Corblin, qui, dans son ouvrage, Indéfini, défini et démonstratif, constructions linguistiques de la référence (Corblin, 1987), propose un parcours très intéressant à travers ces trois désignateurs, tout en ébauchant l'importance de ces opérations linguistiques de reprise dans la construction de chaînes de référence dans le discours. Cet aspect, il le développera plus tard dans Les formes de reprise dans le discours, anaphores et chaînes de référence (Corblin, 1995), où il s'intéresse également à ces formes capables d'assurer un effet de représentation et une certaine cohérence sémantique. Pour cet auteur, on peut établir une claire distinction entre désignation rigide -nom propre et pronom personnel - à éviction totale, et désignation contingente, constituée par des descriptions « identifiantes» de groupes nominaux anaphoriques, des démonstratifs et des indéfinis, qui assure un effet de profusion. 
Puis je me suis retourné et j'ai vu la plaine, cette espèce de gouffre sans limite où plongeaient mes petits chaque soir, le spectacle ne me souleva pas de joie comme il faisait quand je le contemplais du village. Les déserts, la mer, la vaste plaine, l'éternité, attirent peut-être surtout, vus des rivages (Roy, 1977: 102).

La décennie de 1960 connaît Yves Thériault chez qui la nature acquiert une dimension mythique. Agaguk (1958) et Ashini (1969), deux de ses romans les plus connus, invitent le lecteur à découvrir la vie des esquimaux en pleine nature dans l'immensité du Grand Nord ${ }^{2}$. En outre, cette période se caractérise par un sentiment de contestation qui éveille la conscience régionale, voire nationale. Le pays est chanté par des poètes comme Paul-Marie Lapointe avec Arbres-Poèmes (1959) ou Jean-Guy Pilon dans Recours au pays (1961), que nous citons à la fin de notre travail. Ils exalteront la nature, ses couleurs et sons, véritables essences du pays. Mais 1960 c'est aussi la Révolution Tranquille et le début d'une prise de conscience et de parole d'un nombre considérable d'écrivains qui vont avoir recours au passé pour reconstruire leur histoire et consolider ainsi leur identité. Le critique Gaétan Gervais rappelait avec justesse que:

L'identité d'une communauté se fonde sur une certaine mémoire de son passé, sur une certaine compréhension historique [...] L'expérience historique résulte d'événements réels, mais le souvenir qu'on en garde se prête à bien des interprétations. La part du subjectif est donc importante dans la définition d'une identité, les faits et les événements étant sans cesse réinterprétés par les communautés (Gervais, 1995 : 166-167).

Cette pensée contribue à comprendre le fait que la représentation de l'espace dans la littérature des années 70 et 80 devint le moyen d'exprimer les sentiments des Canadiens envers ce vaste pays qui devait devenir leur patrie, mais aussi de réécrire leur propre histoire vis-à-vis de l'espace. Parmi ces romanciers qui vont retourner en arrière pour retracer un passé et une mémoire collective qui risquait de se perdre, nous portons notre intérêt sur l'Acadienne Antonine Maillet qui dans les années 70 commence un projet de récupération des origines mythiques de son Acadie natale avec des romans comme Pointe-aux-Coques (1972), Les Cordes-debois (1977) ou Pélagie-la-Charette (1979). En continuant la ligne de Gabrielle Roy, elle dépeindra les grands espaces naturels, ses bois, collines et bien sûr la mer, comme des intermédiaires entre le passé et le présent. On assiste à la célébration du milieu (Simpson-Housley et Norcliffe, 1992: 149) à travers des descriptions qui suscitent chez le percepteur des sentiments d'appartenance et de reconnaissance de soi-même:

... ce petit village, tout entouré d'eau et de prés verts, qui était pour moi toute l'Acadie (Maillet, 1972: 14).

2 Dans son article «Bibliographie. Comptes rendus bibliographiques» paru dans Revue de l'Université d'Ottawa, vol. XXXII, $\mathrm{n}^{\circ}$ 3, Gaston Rioux signalait : "Ashini, c'est la fraîcheur des sousbois, la liberté des grands espaces, la poésie de l'homme qui sait écouter le vent dans les feuilles, le cri de l'oiseau dans la ramure, le doux murmure du ruisseau à travers les bois » (Rioux, 1962: 364). 
... je m'efforçais d'oublier les années et le village de là-bas, et mes agrès de pêche, et la mer... le fracas des vagues contre la proue, le grondement de la mer au large, le cri des pêcheurs qui s'appellent d'une barque à l'autre... ces matins de juin où presque tout le village s'ébranlait vers la côte... les cris sauvages des oiseaux de mer (Maillet, 1972: 17-18).

La pêche et par extension la mer se transforment en un espace qu'il faut accepter et assimiler pour pouvoir se sentir «Acadien»:

Leurs pères avaient tous pêchés. Et cela remontait loin. Plus de deux siècles sur les côtes acadiennes; et là-bas en Bretagne avant la fondation de la colonie. Sans compter cet exil, ce fameux dérangement de 1755, cette grande aventure. Les Acadiens avaient connu de quoi, à travers leur histoire, se rentrer toute la mer dans les veines (Maillet, 1972: 89).

\section{La dérive énonciative autour des grands espaces dans Chroniques du Nouvel- Ontario d'Hélène Brodeur.}

Pourtant, Antonine Maillet n'est pas la seule à avoir fait de ses descriptions spatiales un lieu d'expression de vécus et de sentiments divers envers ces terres. En effet, les grands espaces canadiens s'étendent au-delà de la mer et incluent les vastes forêts de l'Ontario, qui trouvent chez Hélène Brodeur ${ }^{3}$ une de ses meilleures évocations lyriques. Cette figure représentative de la littérature franco-ontarienne des années 80, publie, entre autres, une trilogie, Chroniques du Nouvel-Ontario, qui présente un grand intérêt non seulement comme un récit rétrospectif de l'histoire des Franco-Ontariens mais aussi comme un témoignage de ce changement de mentalité vis-à-vis d'une réalité spatiale, l'Ontario, qui évolue dans sa façon d'être appréhendé. La composition triptyque de cet ouvrage qui part de 1913 et arrive jusqu'en 1968 nous permet de récapituler ce qui a été dit jusqu'à présent en rapport avec l'expérience de l'espace. Le parcours des fragments descriptifs tirés des trois volets de cette trilogie devient alors un excellent moyen d'exemplifier cette transformation perceptive déjà signalée, alors qu'il nous fournit l'occasion de comprendre le passage de «cette terre» ou «cette immensité» du premier tome vers un emploi de désignations rigides comme «l'Ontario» et même d'expressions «identifiantes» du type «sa patrie», que nous retrouvons dans le dernier livre, reflets d'autre part d'une acceptation de l'espace environnant comme un élément constitutif de l'identité de cette communauté.

La quête d'Alexandre, apparaît comme le premier chaînon des Chroniques $d u$ Nouvel-Ontario; ce récit retrace l'histoire de la région de l'Ontario depuis 1913 jusqu'en 1916, à travers les avatars des personnages, pionniers arrivés au Canada

\footnotetext{
${ }^{3}$ Hélène Brodeur (Saint-Léon-de-Val-Racine, 1923 - Ottawa, 2010) s'impose comme une des voix au féminin en Ontario. Professeur, pigiste et fonctionnaire au gouvernement fédéral, sa trajectoire littéraire comprend non seulement les Chroniques du Nouvel-Ontario (1986 pour les éditions Prise de parole), mais aussi deux autres romans - L'Hermitage (1996) et Marie-Julie (2001); proche de l'autobiographie fictionnelle, son dernier récit porte un regard sur le passé et retrace la vie de la grandmère de l'auteure, dont il fait revivre l'époque (fin du XIXe siècle et début du XXe siècle). La trilogie qui nous occupe fut publiée dans les années 80 et lui a valu à l'écrivaine de nombreux prix, tels le prix Champlain en 1982, le prix Nouvel-Ontario en 1984 et le prix du journal Le Droit en 1985.
} 
dans le désir de bâtir un nouveau pays. Alexandre Sellier, jeune séminariste québécois, part dans le Nord dans le but de retrouver quelque piste de son frère disparu lors du terrible incendie de 1911, occasion qui lui permet de découvrir les grands espaces du nord et de rencontrer de nombreux colons tels que le docteur O'Grady, Cliff Murchison, Eugène Marchessault ou la jeune mariée Rose Brent, arrivée au Canada pour entamer une nouvelle vie et avec qui il maintiendra une relation amoureuse. La perception qu'ont tous ces personnages du même milieu qui les entoure est bien différente. L'abondance de descriptions laisse percevoir une structure binaire de l'espace qui révèle des vécus opposés d'une même réalité spatiale et qui nous évoquent bien sûr les écrits de L. Hémon. Autrement, comment expliquer une perception si différente de l'espace chez Alexandre et chez Rose Brent:

Il [Alexandre] songea que s'il était possible de survoler ce pays dans les machines volantes dont on commençait à parler, on serait ébloui par toutes ces surfaces étincelantes reflétant le soleil, et que la forêt sombre serait comme les cieux étoilés de la nuit, semés de feux sans nombres, reliés comme les galaxies, comme la voie lactée, par les grandes rivières qui sillonnent à l'infini ces immenses étendues (Brodeur, 1985: 53).

... installée dans le train qui se dirigeait vers le Nord elle [Rose] s'efforçait en vain de combattre l'angoisse qui montait sourdement en elle. Depuis son départ de Halifax elle se voyait emportée à toute vitesse dans ce paysage inhumain et apparemment sans limite où les hauts conifères semblaient s'avancer en rangs serrés pour repousser l'intrus qui oserait vouloir y pénétrer. Encore, dans les vallées du Saint Laurent, elle avait vu des villages, un fleuve grand comme la mer, des essences d'arbres familiers comme l'orme et le chêne. Mais maintenant, tout lui semblait étranger. (Brodeur, 1985: 141).

Dans le dernier passage reproduit, nous retrouvons un riche univers descriptif où la vitre du train devient un «topos médiateur» entre un espace fermé, l'intérieur du train, confortable et sans danger et un espace ouvert, immense, défilant dans un mouvement de parcours qui permet l'appréhension de la nouvelle réalité. Le regard de Rose cherche la stabilité, les référents européens, plus civilisés et intimes. D'ailleurs, ce même regard déclenchera quelques pages plus tard un nouveau topos. Cette fois-ci, il s'agît d'une fenêtre d'où ranger et organiser l'environnement spatial en fonction de cette conception binaire dont on a déjà parlé, à savoir, deux cellules qui vont à l'encontre l'une de l'autre: un univers clos, seul indice de vie face à un dur et long hiver blanc, pareil à ce «désert glacé» où Rose se sent perdue: Perdue, elle était perdue dans ce désert glacé. Elle frissonna et machinalement refit le geste qu'il fallait sans cesse répéter: elle ouvrit la porte du poêle et remis du bois sur le feu (Brodeur, 1985: 194).

La perception de l'espace qui s'ensuit des expressions linguistiques employées par le pionnier Eugène Marchessault (formules démonstratif $+\mathrm{N}+$ adjectif) confirme aussi cette continuité de référence, mais avec une claire rupture de point de vue. Maintenant, l'Ontario est saisi comme un endroit haïssable qui suscite un grand malaise: Ce maudit pays a eu sa vie, il n'aura pas son corps (Brodeur, 1985: 193) ou Maudit pays d'enfant de chienne! Si la terre a un trou c'est icitte, dret icitte! (Brodeur, 1985: 275). 
À la suite de ces exemples, nous pouvons distinguer l'existence de deux groupes de personnages qui manifestent linguistiquement leur rapport aux grands espaces de façon antagonique. Au premier groupe constitué par Alexandre, le docteur O'Grady et Cliff Murchison, correspond une vision de l'Ontario comme un milieu naturel, sans doute immense, mais où l'enracinement est possible à travers une acceptation et adaptation progressive aux grands espaces comme élément identitaire. Tandis qu'un deuxième groupe représenté par Eugène Marchessault et auquel appartient, entre autres, Rose Brent exprime sa vision de cette terre inconnue au moyen de constructions démonstratives où les adjectifs témoignent de ce sentiment négatif de refus, de malaise et de lutte contre un espace naturel sauvage et cruel.

Le cas de Rose Brent est sans doute le plus significatif, car les comparaisons continuelles qu'elle ne cesse d'établir entre ce paysage qui l'engloutit et son Angleterre natale où tout semble civilisé, même les distances, ne font que refléter l'imaginaire d'un grand nombre de pionniers européens qui avaient voyagé au Canada, portés par une vision utopique: elle était dans un pays neuf où tout était possible (Brodeur, 1985: 149); mais aussi, munis de leurs référents spatiaux européens : enfin quelque chose qui cadrait avec les images familières et attendues (Brodeur, 1985: 146), avait-elle exclamé à la vue de la grande ferme de Cliff Murchison.

Entre l'aube et le jour, deuxième tome de la trilogie, nous situe en 1930 et trois nouveaux personnages assistent aux successives transformations politiques et sociales qui conduisent le pays vers l'industrialisation. Il s'agit de Rose-Delima, fille des Marchessault, de Donald, né de l'amour entre Rose Brent et Alexandre et de Jean-Pierre Debrettigny. Le train et la voiture réduisent les distances, ce qui déclenche une nouvelle perception de la réalité spatiale. Néanmoins, G. Sénécal rappelait que contigüité et proximité se dilatent dans un environnement technologique qui réduit à néant le critère de distance. Du moment que tombe la contrainte spatiale le triomphe de l'individu vis-à-vis de la communauté territoriale semble total (Sénécal, 1992: 29). À cette transformation de l'imaginaire spatial s'ajoute une réduction considérable des descriptions justifiée par un décor déjà décrit et en procès d'évolution, proche de ce triomphe de l'individu vis-à-vis de l'espace défendu par Sénécal. Mais il existe tout de même quelques fragments qui attirent notre attention. En effet, les deux passages reproduits par la suite, montrent la progression de la représentation linguistique de l'espace. Les constructions démonstratives laissent place à des formes «le $+\mathrm{N}$ », sans adjectif, et même à un emploi du possessif très significatif «sa patrie». La connotation négative de l'emploi du «ce/cette» et d'adjectifs comme «inhumain» ou «maudite» entre autres, se perd et par extension les reclassements continuels. L'Ontario devient désormais le pays de cette génération et une acceptation totale de l'espace comme élément définisseur de l'identité est annoncée grâce à cet emploi du possessif:

Par la fenêtre du train, une femme encore jeune regardait défiler le paysage qu'elle n'avait pas revu depuis près de vingt ans... elle avait tout à coup éprouvé un grand désir de revoir le pays de son adolescence, celui où elle avait connu son premier, son seul amour (Brodeur, 1986a: 131). 
Lorsque la gare disparut elle s'installa près de la fenêtre et se mit à regarder le paysage familier qui fuyait à vitesse accélérée sous ses yeux et qu'elle quittait maintenant. [...] C'était le pays de l'espoir viscéral, où une espèce prépare la voie à une autre. C'était sa patrie. Elle y était née, elle avait grandi dans ce pays austère qui enseignait le courage et l'ingénuité, forçant chaque jour ses enfants à donner leur pleine mesure s'ils devaient survivre (Brodeur, 1986a: 233).

Le dernier discours d'Eugène Marchessault renforce la «mort» de cette attitude et vision de l'espace propre à toute une génération arrivée dans ces grands espaces pour se frayer un chemin en les «combattant», mais sans remporter «la victoire finale»:

Pour la première fois depuis 1916, le désespoir avait provoqué ce cri du cœur: "Si la terre a un trou de cul, c'est icitte, drette icitte", expression qui lui était souvent montée aux lèvres par la suite. [...] Cinq jours plus tard, Eugène décédait. Le rude pays où, jeune homme, il était venu planter ses racines, cet adversaire qu'il avait combattu pendant un quart de siècle, avait emporté la victoire finale (Brodeur, 1986a: 128).

Et, à mi-chemin entre cette dichotomie refus/acceptation des grands espaces se situent les paroles de Jean-Pierre dont l'indécision annonce un renouvellement perceptif, dynamique évolutive qui se consolide dans Les routes incertaines:

Tandis que moi, je n'ai même pas la liberté de partir, fit Jean-Pierre amèrement... S'il se laissait gagner, il se trouverait marié sans aucun espoir de jamais se libérer de ce milieu (Brodeur, 1986a: 201).

Il était toujours heureux de retrouver, son foyer, ses bois et son chien (Brodeur, 1986a: 89).

Les routes incertaines, troisième chaînon de la trilogie, nous porte de 1938 jusqu'en 1968. La jeune génération d'Entre l'aube et le jour ne cesse d'avancer au rythme des temps modernes. Le milieu rural cède définitivement la place aux grandes villes du Sud, et devient cet endroit auquel pouvoir toujours retourner, refuge mythique qui confirme ce rapport intertextuel avec les romans des grands espaces des années 60 . Les références à l'espace acquièrent cette fois-ci une éviction totale, étant «l'Ontario» et «le pays», les formules les plus utilisées par le narrateur; alors que Rose-Delima et Jean-Pierre énoncent ainsi leur identité spatiale: Tu sais, nous les gens du Nord, on a besoin de solitude, de nature et d'espace (Brodeur, 1986b: 126) ou Buvons à notre arrivée au seuil du royaume du Nord. [...] Je reviens chez moi (Brodeur, 1986b: 150).

En ce qui concerne le vieux Alexandre, le lecteur retrouve le passage reproduit ci-dessous:

Il rentrerait finir ses jours dans sa patrie. Il accomplirait ce pèlerinage auquel il avait déjà songé: il retournerait en Ontario-nord.[...] Dans la fraîcheur du matin, son canot glisserait sur les flots calmes et sombres de la rivière Glashini, tandis que la forêt retentirait des chants des oiseaux. Peut-être verrait-il l'orignal majestueux broutant les herbes aquatiques des rives, ou le chevreuil nerveux s'abreuver aux eaux de la rivière.

Sur cette agréable pensée, il s'endormit paisiblement (Brodeur, 1986b: 222).

Alexandre, qui avait quitté «ses Cantons de l'Est» pour pénétrer dans «cette terre inconnue», souhaite à présent rentrer finir ses jours dans «sa patrie». L'Ontario de 
1913 n'est plus l'Ontario de 1968. La perception et le vécu de cet espace ont donc forcément changé, ce qui justifie l'évolution de la désignation linguistique de cet espace.

À travers l'étude exposée de la présence des grands espaces dans la littérature franco-canadienne on a voulu rendre compte de l'importance du facteur spatial dans la construction de l'identité d'une communauté. En retraçant l'évolution du roman acadien, Melvin Gallant, insistait sur le fait que pour qu'une littérature accède à un statut universel, on devrait commencer par parler de soi, du milieu dans lequel on vit, et des particularités de ce milieu. La littérature d'une collectivité ne pourra évoluer que dans la mesure où elle est en accord avec l'âme et la culture de cette collectivité (Tessier, Vaillancourt, 1987: 79). Ainsi, les exemples analysés cherchaient surtout à témoigner de l'importance des descriptions spatiales dans la littérature canadienne comme véhicules d'expression de ce processus de construction identitaire, dont les multiples faces incluent également l'acceptation d'un milieu spatial en tant que patrie. L'approche détaillée de certains passages de la trilogie d'Hélène Brodeur a offert le moyen d'évoquer non seulement cette évolution identitaire en rapport avec l'espace, mais aussi, d'établir et de défendre d'une certaine façon, un rapport intertextuel entre les trois récits et les différentes étapes littéraires présentées au début de notre réflexion. Ainsi, l'on pourrait mettre en rapport les descriptions du premier volume et la conception de la nature présente dans les écrits des années 1900 à 1930. Dans le deuxième volet de la trilogie on assisterait à une exaltation de la ville comme milieu confronté aux grands espaces canadiens que des auteurs des années 40-50 avaient déjà suggérés dans leurs récits; tandis qu'entre les passages descriptifs du troisième tome et les écrits des années 60 , il existe une même représentation de la nature comme un endroit presque mythique, voire un élément constitutif d'une identité toujours en quête.

Nous invitons en dernier lieu à relire ces quelques vers du poète canadien JeanGuy Pilon (Pilon, 1961) qui illustrent, à notre avis, cette idée d'un espace vécu et exprimé:

Comment réussir à dompter les espaces et les saisons, la forêt et le froid? Comment y reconnaître mon visage?

Nous sommes à la naissance d'un pays à reconnaître.

Vient un jour où chaque homme rencontre son pays et lui dit oui à jamais. 


\section{RÉFÉRENCES BIBLIOGRAPHIQUES}

ANOLL, L. FERNÁNDEZ, C., DE LA TORRE, E. (2006): Literaturas francófonas de América y Europa, Madrid, Síntesis.

BORDESSA, R. (1992): «Moral frames for landscape in Canadian literature» in Simpson-Housley and Glen Norcliffe, (eds.), A few acres of snow. Literary and artistic images of Canada, Toronto\&Oxford, Dundurn Press, 58-70.

BRODEUR, H. (1985): La Quête d'Alexandre, Chroniques du Nouvel-Ontario, Sudbury, Prise de Parole.

(1986a): Entre l'aube et le jour, Chroniques du Nouvel-Ontario, Sudbury, Prise de Parole.

(1986b): Les Routes incertaines, Chroniques du Nouvel-Ontario, Sudbury, Prise de Parole.

CORBLIN, F. (1983): «Les désignateurs dans les romans » in Poétiques, 54, 199-211.

COURVILlE, S. SÉGUIN, N. (dir.). (1995): Espace et culture, Québec, Presses de l'Université Laval.

CUBEDDU, S. (2006): «L'américanité québécoise» in Textes et documents pour la classe. Littératures francophones, $\mathrm{n}^{\circ}$ 912, 20-21.

GERVAIS, G. (1995): «Aux origines de l'identité franco-ontarienne» in Cahiers Charlevoix 1. Études franco-ontariennes, Sudbury, Prise de Parole, 125-168.

GILBERT, A. (1986): «L'analyse de contenu des discours géographiques: une méthode» in Le Géographe Canadien, 30 (1), 13-25.

GUILLAUME, S. LERAT, Ch. PICCIONE, M.L. (dir.) (1996): L'espace canadien et ses représentations, Talence, Éditions de la maison des sciences de l'homme d'Aquitaine.

GRADY, W. (1992): Treasures of the place, three centuries of Nature writing, Vanvouver/Toronto, Douglas \& McIntyre.

HAMON, PH., (1993): Du descriptif, Paris, Hachette Supérieur.

HÉMON, L., (1954): Maria Chapdelaine, Paris, Grasset.

HOTTE, L. et MELANÇON, J. (dir.) (2005): Thèmes et variations. Regards sur la littérature franco-ontarienne, Sudbury, Prise de Parole.

HUGHES, T. (1987): Gabrielle Roy et Margaret Laurence: deux chemins, une recherche, Saint-Boniface, Les éditions du blé. Coll. Soleil.

LEMIRE, M. (1996): «L'appel des grands espaces » in GUILLAUME, S. LERAT, Ch. PICCIONE, M.L. (dir.) (1996): L'espace canadien et ses représentations, Talence, Éditions de la maison des sciences de l'homme d'Aquitaine.

MAILlET, A. (1972): Pointe-aux-Coques, Montréal, Leméac.

MANGADA, B. (2003): Hélène Brodeur, Ottawa, Éditions David.

PILON, J.-G. (1961): Recours au pays, Montréal, Ed. L’Hexagone.

RIOUX, G. (1962): «Bibliographie. Comptes rendus bibliographiques» in Revue de l'Université d'Ottawa, vol. XXXII, $\mathrm{n}^{\circ}$ 3, juillet-septembre, 363-368.

ROY, G., (1966): La Route d'Altamont, Montréal, HMH.

(1977): Ces enfants de ma vie, Montréal, Alain Stanké.

(1980): La Petite Poule d'Eau, Montréal, Éditions internationales, Alain Stanké.

SÉNÉCAL, G. (1992): «Aspects de l'imaginaire spatial: identité ou fin des territoires?» in Annales de Géographie, 563, 28-42. 
SIMPSON-HOUSLEY, P., \& NORCLIFFE, G., (1992): A few acres of snow. Literary and artistic images of Canada, Toronto\&Oxford, Dundurn Press.

TESSIER, J., VAILLANCOURT, P.-L., (1987): Les autres littératures d'expression française en Amérique du Nord, Ottawa, Presses de l'Université d'Ottawa, Cahiers du CRCCF.

TREPANIER, E., (1996): «La peinture québécoise d'entre-deux-guerres» in GUILLAUME, S. LERAT, Ch. PICCIONE, M.L. (dir.): L'espace canadien et ses représentations, Talence, Éditions de la maison des sciences de l'homme d'Aquitaine, 43-53. 